\title{
Evaluation of Cervical Posture Improvement of Children with Cerebral Palsy after Physical Therapy with a HCI Based on Head Movements and Serious Videogames
}

\author{
Miguel A. Velasco ${ }^{1}$, Rafael Raya ${ }^{1,2}$, Luca Muzzioli $^{3}$, Daniela Morelli ${ }^{3}$, Marco Iosa $^{3}$, \\ Febo Cincotti $^{3}$ and Eduardo Rocon ${ }^{1}$ \\ ${ }^{1}$ Centro de Automática y Robótica CAR, UPM-CSIC, Ctra. Campo Real Km 0.2, 28500 Ar- \\ ganda del Rey, Madrid, Spain \\ miguel.velasco@csic.es \\ ${ }^{2}$ Department of Information Technology, Universidad CEU San Pablo, Spain. \\ ${ }^{3}$ Fondazione Santa Lucia FSL, Via Ardeatina, 306, 00142 Rome, Italy
}

\begin{abstract}
This paper presents the preliminary results of a novel rehabilitation therapy for cervical and trunk control of children with cerebral palsy $(\mathrm{CP})$. The therapy is based on the use of an inertial sensor that will be used to control a set of serious videogames with movements of the head. Ten users with $\mathrm{CP}$ participated in the study, in the experimental and control groups. Ten sessions of therapy provided improvements in head and trunk control that were higher in the experimental group for Visual Analogue Scale (VAS), Goal Attainment Scaling (GAS) and Trunk Control Measurement Scale (TCMS). Significant differences ( $27 \%$ vs. $2 \%$ of percentage improvement) were found between the experimental and control groups for TCMS ( $\mathrm{p}<0.05)$. The kinematic assessment shows that there are some improvements in active and passive range of motion, but no significant differences were found pre- and after-therapy. This new strategy, together with traditional rehabilitation therapies, could allow the child to reach maximum levels of function in the trunk and cervical regions.
\end{abstract}

Keywords: cerebral palsy·cervical posture-inertial sensor·serious games

\section{$1 \quad$ Introduction}

Cerebral palsy (CP) is a disorder of posture and movement due to a defect or lesion in the immature brain [1]. CP affects between 2 to 3 per 1000 live-births, reported for the European registers by the Surveillance of Cerebral Palsy European Network (SCPE) [2], and there is a prevalence of three to four per 1000 among school-age children in the USA [3]. CP is the most common cause of permanent serious physical disability in childhood, and the prospect of survival in children with severe level of impairment has increased in recent years, [4]. CP is often associated to sensory deficits, cognition impairments, communication and motor disabilities, behavior issues, seizure disorder, pain and secondary musculoskeletal problems. CP can be classified according to different criteria: the distribution of the deficits, the gross motor func- 
tion, the predominant abnormality, and other systems. The "Surveillance of Cerebral Palsy in Europe (SCPE): a collaboration of cerebral palsy surveys and registers" presented a consensus on the definition, classification and description of $\mathrm{CP}[5,6]$.

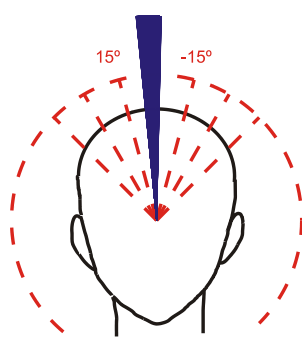

$\mathrm{Y} \longleftarrow \oiint_{\mathrm{X}}^{\mathrm{Z}}$

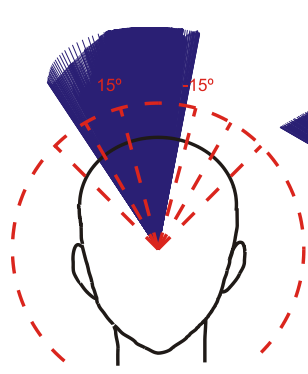

$\mathrm{N^{2 }}$

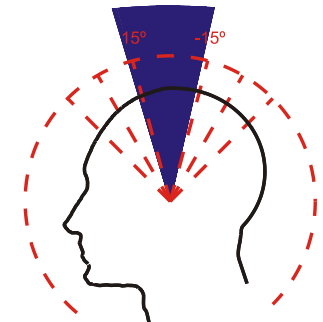

$\stackrel{P}{z}^{2}$
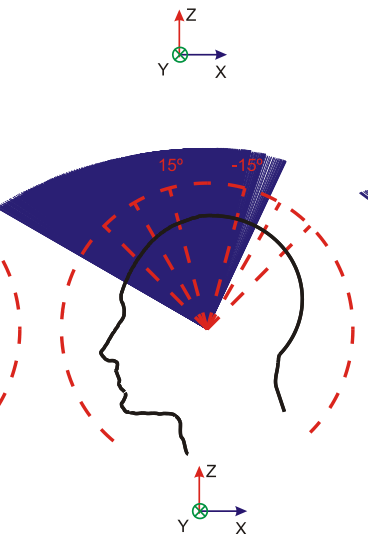

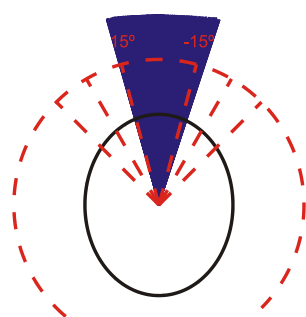

y $\hat{\oint}_{z}^{x}$

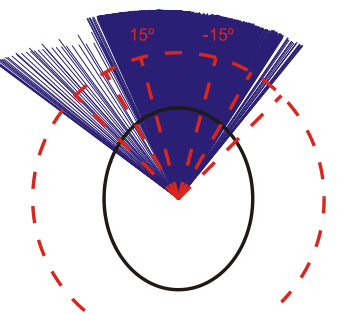

$y \oint_{z}^{x}$

Fig. 1. Angular orientations in frontal, sagittal and transverse planes measured with the ENLAZA interface. Above, an individual without motor disorders; below, one with $\mathrm{CP}$.

Poor head and trunk control in CP produce limitations beyond function. In hypotonic CP infants (as in Fig. 1) they can be so severe that the infant may experience difficulty breathing or swallowing since effective oral functioning for feeding begins with attaining better head stability to improve jaw control [7]. Moreover, the head is responsible for the directional orientation of the special senses and its movements are influenced by the information these provide. It is not unexpected that certain disorders of the special senses may lead to unusual head movements and that disorders of head movement may force unusual conditions upon the special senses [8].

Posture improvement is important not only because of functional reasons, but also to improve some secondary conditions related with health and social interaction. Kramer et al. [9] compared the semi-prone (SP) and sitting (SIT) training positions with respect to head control in children with $\mathrm{CP}$, before and after 5 weeks biofeedback training using a head position trainer (HPT). They concluded that biofeedback training with a HPT can be effective in either the SIT or the SP positions, with improvement lasting at least 16 weeks after training is discontinued. 
A program was recently developed to promote adaptive responses and upright head position in students with multiple disabilities (cerebropathy and spastic tetraparesis and their head tended to be tilted forward) through the use of micro-switch clusters (i.e., combinations of two micro-switches) during a stimulation period [10]. The study consisting in measuring their actual level of head control, that is, the length of time they kept the upright head position. The five students exposed to the program showed a significant increase in adaptive responses performed with head upright. A recent research investigated the head position correction using a commercial inertial product. Two subjects with severe multiple disabilities with spastic quadriplegic cerebral palsy with limited trunk and head control participated. Results showed significantly increased their time duration of maintaining upright head position to obtain the desired environmental stimulation [11].

This study is presented as a proof of concept of a rehabilitation therapy for the improvement of head and trunk posture in children with CP based on active head exercises performed through serious videogames that will be accessed with an interface based on inertial technology. We aim to develop evidence-based criteria for the integration of these exercises into the traditional therapies and to determine their role in maximizing head control in children with $\mathrm{CP}$. We hypothesize that the user can improve his/her head posture by using the ENLAZA interface based on the neuroplasticity and the capacity to learn new motor skills.

\section{Background}

\subsection{Assessment of Cerebral Palsy}

When assessing people with $\mathrm{CP}$, many factors and the symptoms need to be monitored. The WHO International Classification of Functioning, Disability and Health (ICF) along with several other recent publications such as the SCPE have sensitized health professionals to the importance of evaluating the functional consequences of different health states. For ambulation, the Gross Motor Function Classification System (GMFCS) [12] has been widely employed internationally to group individuals with $\mathrm{CP}$ into one of five levels based on functional mobility or activity limitation. So has the bimanual fine motor function system BFMF [13], or, in prospective studies, the Manual Ability Classification System MACS [14].

Most methods are subjective measures that classify the motor involvement on the basis of functional abilities. In milder cases, the assessment and conclusions may vary by the subjective examinations of various professionals. Therefore, a combination of significant motor developmental delay and abnormalities in the neurologic examination is required to make the diagnosis. A promising approach is the use of normal and abnormal general movement patterns. This method appears to have high sensitivity and specificity for the diagnosis of CP [15,16]. Motion sensing, by means of MEMS inertial sensors a real scientific breakthrough in the medical field, where there is a need for small ambulatory sensor systems for measuring the kinematics of body segments [17]. As a result, inertial sensors have been chosen for different applications focused on people with motor disorders, such as the evaluation of clinical spasticity 
assessment (by measuring the range of motion) [18] and the quantification of standing balance by assessing displacement of the center of mass in CP [19] and clinical assessment of tremor in Parkinson [20].
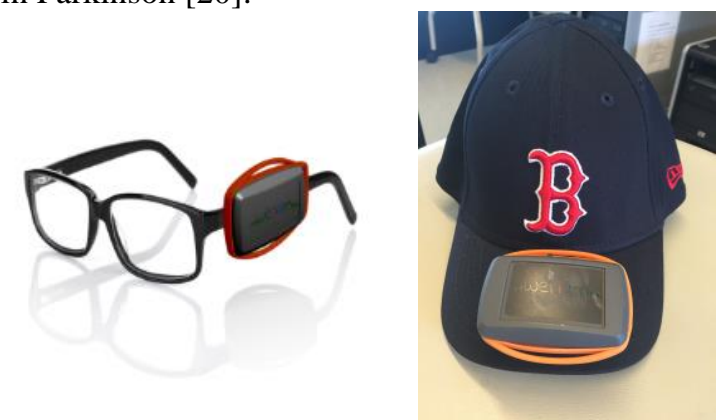

Fig. 2. The ENLAZA interface (Werium Solutions S.L., Spain)

\subsection{Therapies and Treatments}

Treatments for CP patients depend on the patient's presentation and range from physical therapy to medication and surgery. They follow these basic principles [21]: (1) emphasis on normalization of the quality of movement; (2) emphasis on functional activities, which focuses on the development of skills necessary for the performance of activities of daily living (ADL). Superior clinical results have been observed in children participating in this intervention [22].

The priorities in the management of $\mathrm{CP}$ are currently moving from traditional strategies focusing on promoting compensation towards new strategies aiming on restore motor function with the increasing evidence of neuroplasticity and motor learning theories. These strategies have a higher impact during early ages because of neuroplasticity, i.e. the ability of the neurons and other human brain cells to reorganize their structure and function after an injury, in response to different external and internal factors, including physical training [23]. Task-oriented therapies aim to improve the movement and the posture of the user with $\mathrm{CP}$ by the repetitive training performing a certain functional task. People with CP frequently show impaired limb, trunk and head control, which affect performances of ADLs. The majority of research in children with CP focuses on assessment and treatment of upper and lower extremities. In contrast, literature on trunk and head control in children with CP is scarce [24].

\section{The ENLAZA Human-Computer Interface (HCI)}

Raya et al. proposed the ENLAZA interface [25], an adapted input device for users with severe motor disorders (especially CP) that cannot use traditional solutions such as mice, joysticks or trackballs to access the computer. ENLAZA allows users to control the cursor of the computer with movements of their heads and consists of a headset with a cap and an inertial measurement unit, IMU, (Werium S.L., Spain) that inte- 
grates a tridimensional (3D) accelerometer, a 3D gyroscope and a 3D magnetometer mounted on a commercial helmet. A calibrated IMU measures 3D acceleration (caused by motion and gravity), 3D angular velocity and 3D earth magnetic field. A data fusion algorithm presented in [26] estimates the IMU orientation and enables measurement of inclination changes less than $1.0^{\circ}$ and $1^{\circ}-2^{\circ}$ heading accuracy.

The user wears the ENLAZA device at the beginning of the experiment. After wearing the device, the calibration procedure starts. This procedure consisted of maintaining the head in front on the computer screen (zero position) and calibrate angular rotation required to reach the horizontal and vertical bounds of the screen. Once calibrated for the first session, it is not necessary to repeat it for the next sessions.

For the purpose of this study, the mouse pointer is controlled with an absolute control, meaning that there is a unique mapping between head orientation and location of the pointer. After a calibration process, all pixels in the screen are reachable for the user's Cervical Range of Motion, CROM. During the calibration, a therapist adjusts the gain of the transfer function that translates the orientation of the head into a location of the pointer on the screen. A Robust Kalman Filter (RKF) [27] was developed to facilitate fine motor control based on the characterization of involuntary movements found in users with cerebral palsy. In addition, the designed software captures data used to assess performance in the task. In particular, the application captures the positions of the mouse pointer and target during the session.

\section{$4 \quad$ Methodology}

Five users with CP from the "Fondazione Santa Lucia" (FSL, Rome, Italy), a center specialized in the treatment of CP participated on this study. The inclusion/exclusion criteria can be found in Table 1. Participants wore ENLAZA and played serious videogames. Six videogames have been specially designed and developed in C\# and the framework .NET 4.0 to be played with the ENLAZA interface, and another set of six commercial off-the-shelf videogames have been adapted to be played with this system. These videogames gather the following characteristics:

Table 1. Inclusion/exclusion criteria

\begin{tabular}{l|l}
\hline Inclusion criteria & Exclusion criteria \\
\hline -Males and females, aged 4-21 years old & -Aggressive or self-injure behavior. \\
-Diagnosed CP and cervical hypotonia or & -Involuntary movements on the head. \\
difficulties on head control & \\
-Cognitive capacity and behavior appro- & -Cervical surgery within the previous 6 \\
priate to understand the tasks and follow & months. \\
simple instruction and active participa- & \\
tion in the study, & \\
-Signed written informed consent by & $\begin{array}{l}\text {-Inability to control the ENLAZA sys- } \\
\text { parents or legal guardian. }\end{array}$ \\
-Medically stable. & $\begin{array}{l}\text { tem during the first testing session. } \\
\text {-Severe visual limitations }\end{array}$ \\
\hline
\end{tabular}


- Fun and systematic.

- Visual and auditory feedback

- Clear objective for the user: Task and duration.

- Different levels of difficulty.

- To use colours or images to represent abstract concepts as time.

Another 5 users with CP were recruited for the control group. They followed the traditional physical and occupational therapy.

\subsection{Assessment of Head Posture}

We propose a kinematic and functional analysis of the improvement hypothesized.

\section{Kinematic assessment.}

We will use ENLAZA to measure (1) the CROM, i.e. flexion-extension, rotations and lateral flexion, during active movements directly performed by the child and (2) the CROM during passive mobilization of the therapist.

\section{Functional assessment.}

The outcome measures to measure the head posture improvement will be:

- Gross Motor Function Measure-88 (GMFM-88). We will use the items 21 and 22 depending on the child's skills [28]. They assess whether a child can lift and maintain his/her head in a vertical position with trunk support by a therapist while sitting.

- Visual Analogue Scale (VAS). VAS is a valid scale that consists of a line of 100 mm separating two labels: $0=$ "No head control" and $10=$ "Perfect head control". We asked parents, children and therapist to put a cross on the line of $100 \mathrm{~mm}$ separating these two labels to indicate on the VAS the level of head control of the children.

- Goal attainment scaling (GAS). GAS allows the therapist to program a desired improvement and to judge if the child achieved it (the fact that the goal is chosen by therapist made this scale very sensitive). Goal 1 will be related to head movement, and goal 2 will be optional and related to choking/swallowing, if the child has daily trouble swallowing.

\subsection{Data Analysis}

Mean and standard deviation have been used for the description of clinical scale scores. Percentage improvement has been evaluated as (post- and pre-value ) / prevalue*100. Non parametric inferential statistical tests have been used: in particular the Wilcoxon signed ranks test was used for within group analyses in order to compare clinical scores at T0 and T1, whereas the Mann-Whitney u-test was used for the be- 
tween group comparisons at T0 and at T1, separately. The alpha level of statistical significant threshold was set at 0.05 for all the analyses.

\section{$5 \quad$ Results}

\subsection{Functional Assessment}

Tables 2 and 3 show the mean clinical scores pre and post intervention. The changes resulted statistically significant in terms of control of the head $(\mathrm{p}=0.034)$, visuomotor control assessed by GAS-score $(\mathrm{p}=0.043)$ and TCMS $(\mathrm{p}=0.042)$. The gross motor functioning slightly improved but the value of $\mathrm{p}$ did not achieve the statistical significant threshold $(\mathrm{p}=0.048)$. The items 21 and 22 of GMFM- 88 remained unaltered both in experimental as well as in control group.

For the control group, statistically significant improvements occurred in terms of head control $(\mathrm{p}=0.046)$ and visuomotor control $(\mathrm{p}=0.042)$, but neither in terms of trunk control $(\mathrm{p}=0.317)$ nor of gross motor functioning $(\mathrm{p}=0.317)$. It implied that the improvement of trunk control was significantly higher in Experimental Group with respect to Control Group (about $+27 \%$ vs. $+2 \%$, respectively, as reported in Table 3 and Fig. 3). The other percentage changes, despite quite higher in experimental group, were not statistically different between the two groups.

Table 2. Clinical scores for Experimental Group (p-value refers to Wilcoxon signed rank test)

\begin{tabular}{l|ccc}
\hline Experimental Group & Pre & Post & p-value \\
\hline VAS & $6.4 \pm 1.1$ & $7.6 \pm 1.3$ & $\mathbf{0 . 0 3 4}$ \\
GAS & $22.8 \pm 0.4$ & $64.3 \pm 3.6$ & $\mathbf{0 . 0 4 3}$ \\
TCMS & $19.4 \pm 14.1$ & $24.2 \pm 17.9$ & $\mathbf{0 . 0 4 2}$ \\
GMFM-88 & $44.4 \pm 23.2$ & $50.2 \pm 27.8$ & 0.068 \\
GMFM-88 Item 21 & $3 \pm 0$ & $3 \pm 0$ & 0.999 \\
GMFM-88 Item 22 & $3 \pm 0$ & $3 \pm 0$ & 0.999 \\
\hline
\end{tabular}

Table 3. Percentage improvements in clinical scores for Experimental vs- Control Group (pvalue refers to Mann Whitney u test)

\begin{tabular}{l|ccc}
\hline Scale & Experimental group & Control group & p-value \\
& & & \\
\hline VAS & $18.9 \pm 6.0 \%$ & $15.2 \pm 9.4 \%$ & 0.690 \\
GAS & $181.3 \pm 17.0 \%$ & $155.6 \pm 27.9 \%$ & 0.222 \\
TCMS & $27.2 \pm 11.5 \%$ & $1.8 \pm 4.1 \%$ & $\mathbf{0 . 0 0 8}$ \\
GMFM-88 & $11.5 \pm 18.7 \%$ & $0.8 \pm 1.77 \%$ & 0.151 \\
\hline
\end{tabular}




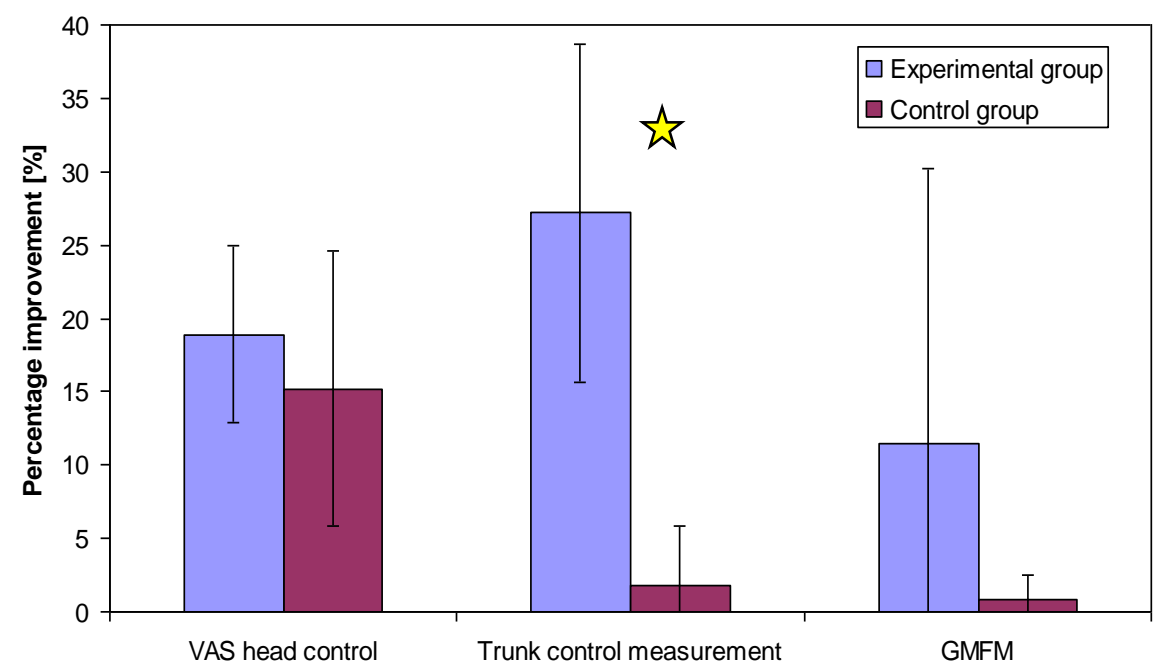

Fig. 3. Mean and standard deviations of percentage improvements in the groups. Stars indicate a statistically significant difference between groups assessed by Man Whitney u test $(\mathrm{p}<0.05)$

\subsection{Kinematic Assessment}

The ranges of motion measured before and after the therapy showed a rise of AROM in all three movements. The percentages of increment were $+20 \%,+38 \%$ and $+85 \%$ in order to achieve $93^{\circ}, 90^{\circ}$ and $145^{\circ}$ for active flexion-extension, lateral flexion and rotation, respectively. Unfortunately, no statistically significant differences were found $(\mathrm{p}=0.18, \mathrm{p}=0.62, \mathrm{p}=0.43$ ).

The passive range of motion, PROM, presented smaller changes: an increment of $+5 \%$ and $+57 \%$ to achieve $77^{\circ}$ and $140^{\circ}$ of passive lateral flexion and rotation. Passive range of motion during flexion experienced a small reduction $(-6 \%)$ and decremented from $86^{\circ}$ to $81^{\circ}$. Once again, no statistical significance was found $(\mathrm{p}=0.43, \mathrm{p}=0.62$, $\mathrm{p}=0.43)$.

\section{Discussion and Conclusion}

We found improvements in four metrics (VAM, GAS, TCMS and GMFM-88) in the experimental group, although they were not significant for GMFM-88. The improvements were generally better for the experimental group and significant differences were found in TCMS between the groups. The percentage of improvement in trunk control is indeed remarkable and shows the potential of this kind biofeedback in rehabilitation therapies.

Despite the lack of statistical significance in the improvements, the values AROM and APROM for flexion, lateral-flexion and extension after 10 work sessions are 
closer to the physiologically normal ROMs, i.e. $90^{\circ}, 160^{\circ}$ and $90^{\circ}$ for flexionextension, lateral flexion and rotation.

We proved a therapy for the rehabilitation of head and trunk motor control with inertial sensors and serious games as a complement to traditional therapies is possible, and that the improvements of this novel therapy are better that those achieved with traditional therapies alone. In future experiments, we will recruit a larger group in a multi-center study in order to look for greater significance in the functional and kinematic evaluation.

Acknolegdements. Authors would like to thank the members and staff in FSL. This work was possible thank to ABC EU Project, CPWALKER Project, INTERPLAY Project and IVANPACE Project, which is funded by Obra Social de Caja Cantabria.

\section{References}

[1] M. Bax, M. Goldstein, P. Rosenbaum, A. Leviton, N. Paneth, B. Dan, B. Jacobsson, and D. Damiano, "Proposed definition and classification of cerebral palsy, April 2005.," Dev. Med. Child Neurol., vol. 47, no. 8, pp. 571$576,2005$.

[2] A. Johnson, N. Perinatal, E. Unit, and O. Road, "Prevalence and characteristics of children with cerebral palsy in Europe.," Dev. Med. Child Neurol., vol. 44, no. 9, pp. 633-40, Sep. 2002.

[3] S. Winter, a. Autry, C. Boyle, and M. Yeargin-Allsopp, "Trends in the Prevalence of Cerebral Palsy in a Population-Based Study," Pediatrics, vol. 110, no. 6, pp. 1220-1225, Dec. 2002.

[4] E. Blair, "Epidemiology of the cerebral palsies," Orthop. Clin. North Am., vol. 41, no. 4, pp. 441-455, 2010.

[5] C. Cans, "Surveillance of cerebral palsy in Europe: a collaboration of cerebral palsy surveys and registers," Dev. Med. Child Neurol., vol. 42, no. 12, pp. 816-824, Feb. 2007.

[6] I. Krägeloh-Mann and C. Cans, "Cerebral palsy update," Brain Dev., vol. 31, no. 7, pp. 537-544, 2009.

[7] F. Redstone and J. F. West, "The importance of postural control for feeding.," Pediatr. Nurs., vol. 30, no. 2, pp. 97-100, 2004.

[8] M. a Gresty and G. M. Halmagyi, "Abnormal head movements.," J. Neurol. Neurosurg. Psychiatry, vol. 42, no. 8, pp. 705-714, 1979.

[9] J. F. Kramer, B. Ashton, and R. Brander, "Training of head control in the sitting and semi-prone positions.," Child. Care. Health Dev., vol. 18, no. 6, pp. 365-76, 1992.

[10] G. E. Lancioni, N. N. Singh, M. F. O’Reilly, J. Sigafoos, R. Didden, D. Oliva, and L. Severini, "Fostering adaptive responses and head control in students with multiple disabilities through a microswitch-based program: follow-up assessment and program revision.," Res. Dev. Disabil., vol. 28, no. 2, pp. 187-96, 2007. 
[11] C.-H. Shih, "Assisting people with disabilities improves their collaborative pointing efficiency through the use of the mouse scroll wheel.," Res. Dev. Disabil., vol. 34, no. 1, pp. 1-10, Jan. 2013.

[12] G. E, R. R. Palisano, P. Rosenbaum, D. Bartlett, and M. Livingstone, "GROSS MOTOR FUNCTION CLASSIFICATION SYSTEM Extendida y Revisada," Child. A Glob. J. Child Res., 2007.

[13] E. Beckung and G. Hagberg, "Correlation between ICIDH handicap code and Gross Motor Function Classification System in children with cerebral palsy.," Dev. Med. Child Neurol., vol. 42, pp. 669-673, 2000.

[14] Eliasson AC, Krumlinde Sundholm L, Rösblad B, Beckung E, Arner M, Öhrvall AM, Rosenbaum P, “The Manual Ability Classification System (MACS) for children with cerebral palsy: scale development and evidence of validity and reliability" 2006.

[15] R. Mac and K. Lecture, “The 1999 Ronnie Mac Keith Lecture,” pp. 836-842, 2001.

[16] J. van der Heide, P. B. Paolicelli, a Boldrini, and G. Cioni, "Kinematic and qualitative analysis of lower-extremity movements in preterm infants with brain lesions.," Phys. Ther., vol. 79, no. 6, pp. 546-557, 1999.

[17] M. Kim, B. H. Kim, and S. Jo, "Quantitative evaluation of a low-cost noninvasive hybrid interface based on EEG and eye movement.," IEEE Trans. Neural Syst. Rehabil. Eng., vol. 23, no. 2, pp. 159-68, 2015.

[18] A. Cutti, A. Cappello, and A. Davalli, "A new technique for compensating the soft tissue artefact at the upper-arm: in vitro validation," J. Mech. ..., vol. 5, no. 2, pp. 1-15, 2005.

[19] H. Ghasemzadeh, R. Jafari, and B. Prabhakaran, “A body sensor network with electromyogram and inertial sensors: Multimodal interpretation of muscular activities," IEEE Trans. Inf. Technol. Biomed., vol. 14, no. 2, pp. 198-206, 2010.

[20] H. P. Jr, M. Hanson, and J. Lach, "A Wearable Inertial Sensing Technology for Clinical Assessment of," Circuits Syst. Conf. BIOCAS 2007, pp. 9-12, 2007.

[21] E. Bower, "The multiply handicapped child," in Rehabilitation Studies Handbook, B. A. Wilson and L. D. McLellan, Eds. Cambridge University Press, 1997, pp. 315-354.

[22] M. Ketelaar, a Vermeer, H. Hart, E. van Petegem-van Beek, and P. J. Helders, "Effects of a functional therapy program on motor abilities of children with cerebral palsy.," Phys. Ther., vol. 81, no. 9, pp. 1534-1545, 2001.

[23] M. L. Aisen, D. Kerkovich, J. Mast, S. Mulroy, T. a L. Wren, R. M. Kay, and S. a. Rethlefsen, "Cerebral palsy: Clinical care and neurological rehabilitation," Lancet Neurol., vol. 10, no. 9, pp. 844-852, 2011.

[24] L. Heyrman, G. Molenaers, K. Desloovere, G. Verheyden, J. De Cat, E. Monbaliu, and H. Feys, "A clinical tool to measure trunk control in children with cerebral palsy: The Trunk Control Measurement Scale.," Res. Dev. 
Disabil., vol. 32, no. 6, pp. 2624-2635, 2011.

[25] R. Raya, R. Ceres, E. Rocon, T. González, and A. Ruiz, "Desarrollo de un interfaz inercial orientado a terapias alternativas en la parálisis cerebral," in Actas de las IV Jornadas Iberoamericanas de Tecnologías de Apoyo a la Discapacidad, 2009, pp. 57-62.

[26] R. Raya, J. O. Roa, E. Rocon, R. Ceres, and J. L. Pons, "Wearable inertial mouse for children with physical and cognitive impairments," Sensors Actuators A Phys., vol. 162, no. 2, pp. 248-259, Aug. 2010.

[27] R. Raya, E. Rocon, J. a Gallego, R. Ceres, and J. L. Pons, “A robust kalman algorithm to facilitate human-computer interaction for people with cerebral palsy, using a new interface based on inertial sensors.," Sensors (Basel)., vol. 12, no. 3, pp. 3049-67, Jan. 2012.

[28] R. Weis, "Gross Motor Function Measure (GMFM-66 and GMFM-88) User's Manual," European Journal of Paediatric Neurology, vol. 8. pp. 111-112, 2004. 